\title{
Two-tuples Linguistic Models and Methods for Target Threat Grading Assessment
}

\author{
Shuqi Wang and Liang Ma* \\ Department of Surface Ship Command, Dalian Naval Academy, Dalian, 116018, China \\ 446982051@qq.com, maliang2014@tom.com
}

Keywords: Target threat grading assessment; Two-tuples linguistics; Combat command and decision; Membership degree; Multiattribute comprehensive assessment; Group sorting decision

\begin{abstract}
The target threat ranking and grading assessment is the premise of the firepower allocation and also is an important part of the combat command and control system. A general model of the target threat grading assessment problem is formulated and the linear weighted comprehensive assessment method is proposed in this paper. Analyzing limitation in determination of target threat grades using the maximum membership degree principle, the target threat grading assessment model and method are proposed by introducing the definition of grade determinant values and the concept of the two-tuples linguistics. The proposed methodology may provide theory basic and intelligent support for combat command and decision and can be easily extended to solve multiattribute/criterion group sorting decision problems.
\end{abstract}

\section{Introduction}

Target Threat Assessment, also known as estimated or Threat judgment, contains the sort of target Threat judgment and the grade of target Threat Assessment two aspects. Target threat assessment is closely related to the battlefield situation assessment, its main task is in accordance with the relevant data by manual intervention infer the enemy targets to the size of the target threat degree, the result will directly affect their deployment and adjustment of fire distribution. Target threat assessment, has been a commander and military experts focus on the problems, because of the war in traditional relatively changes its complexity, scale, speed and technical content is low, rely on the commander and the military expert knowledge and experience can finish the work of target threat judgment. But in today's information-based war, with only a commander and military experts knowledge and experience to judge and decision-making have been unable to meet the requirements.

On the research of target threat assessment at home and abroad a lot, especially in the theoretical model and the method of research on different [1]. Due to the influence of target threat degree of many factors, including the target type, distance, height, speed, course short, interference ability, quantity, etc., and between these factors influence often conflict each other, not commensurability, therefore target threat assessment in essence can be summarized as a kind of multiple attribute or multi-factor comprehensive evaluation problems.

Some research on the grade of target threat sequencing and evaluation as the same kind of problem to solve [2] [3]. In fact, after the comprehensive value of target threat was obtained threat level is needed according to the setting threshold, the comprehensive value of target threat judgment whether meet the threshold condition to determine the grade of target threat, it has a direct influence on and the fire distribution. This article explores the binary semantic model and the method for grade of target threat for processing. 


\section{Grade of Target Threat Evaluation Mathematical Description}

There is a group of target $T_{j}(j=1,2, \cdots, n)$ sets, a threat factor $T=\left\{T_{1}, T_{2}, \cdots, T_{n}\right\}$ threat for subset; By taking $e_{k}(k=1,2, \cdots, h)$ said the threat leve $F=\left\{f_{1}, f_{2}, \cdots, f_{m}\right\}$, and regulation. The first threat level with a threat level than the first one is strong, all set a level of threat level $E=\left\{e_{1}, e_{2}, \cdots, e_{h}\right\}$.

Target $T_{j}$ threat level about $f_{i}$ the characteristics of the value of $y_{i j k}=f_{i k}\left(T_{j}\right)$. Can be visually represented as in matrix form $\boldsymbol{Y}_{j}=\left(y_{i j k}\right)_{m \times h}$, referred to $T_{j}$ as the target's threat level characteristic value of matrix. Said in the first line, the goal $T_{j}$ of all the eigenvalues of the threat $f_{i}$ level of threat factor, and the first column represents the target all the eigenvalues of the threat factors of threat level, remember to $\boldsymbol{y}_{j}=\left(y_{1 j k}, y_{2 j k}, \cdots, y_{m j k}\right)^{\mathrm{T}}(k=1,2, \cdots, h)$.

The characteristic values for grade of target threat $y_{i j k}(i=1,2, \cdots, m ; k=1,2, \cdots, h)$ Type is usually not the same, need to do to its standardization transformation, makes:

$y_{i j k} \in \mathrm{R} \mapsto \mu_{i j k}=\phi_{1}\left(y_{i j k}\right) \in[0,1]$

In this type, $\phi_{1}$ is threat factor level is characteristic value standardization method or transform operator, according to the actual selection; $\mathrm{R}$ Is a real number set.

Use type (1) can be normalized $\mathbf{Y}_{j}$ referred to as the standardization of the target threat factor level characteristic value of matrix $\boldsymbol{\mu}_{j}=\left(\mu_{i j k}\right)_{m \times h}$. Similarly, expressed in $\boldsymbol{\mu}_{j k}=\left(\mu_{1 j k}, \mu_{2 j k}, \cdots, \mu_{m j k}\right)^{\mathrm{T}}$ all about the standardization of the threat factor eigenvalues $T_{j}$.

Threat level division is a fuzzy concept, the subjectivity of the larger depends on the defenders don't performance and decision thought, friend or foe of battlefield, and many other factors, therefore, $\boldsymbol{\mu}_{j}$ is considered to be a and a fuzzy relation between $F$ and $E$, which is about the membership degree of threat level $\mu_{i j k}, \boldsymbol{\mu}_{j k}$ is about all the threat factor the membership degree of the vector. $\boldsymbol{\mu}_{j}$ Can also be called of the level of threat factor evaluation vector, referred to as the level of threat factor evaluation matrix $\boldsymbol{\mu}_{j}$.

According to some kind of evaluation method for grade of target threat or rally operator $^{\phi}:[0,1]^{m+h} \rightarrow\left[0,1^{h}\right]$, can get the target $T_{j}(j=1,2, \cdots, n)$ for all the threat level of comprehensive membership $e_{k}$ vector is:

$$
\boldsymbol{U}_{j}=\phi\left(\omega, \boldsymbol{\mu}_{j}\right)
$$

Type, $\boldsymbol{\omega}=\left(\omega_{1}, \omega_{2}, \cdots, \omega_{m}\right)^{\mathrm{T}}$ is a threat factor weight vector. $\boldsymbol{U}_{j}=\left(u_{j 1}, u_{j 2}, \cdots, u_{j h}\right)$ is a membership degree vector. $u_{j k}=\phi\left(\boldsymbol{\omega}, \boldsymbol{\mu}_{j k}\right) \in[0,1](k=1,2, \cdots, h)$ and $\sum_{k=1}^{h} u_{j k}=1$

The number of target threat level comprehensive membership vector visually represented as $\boldsymbol{U}=\left(u_{j k}\right)_{n \times h}$, in matrix form, referred to as the comprehensive membership degree matrix for grade of target threat. In type (1) a relatively simple linear weighted comprehensive evaluation model of the specific form is commonly used:

$$
\boldsymbol{U}_{j}=\left(\frac{\boldsymbol{\omega}^{\mathrm{T}} \boldsymbol{\mu}_{j k}}{\sum_{k=1}^{h} \boldsymbol{\omega}^{\mathrm{T}} \boldsymbol{\mu}_{j k}}\right)_{1 \times h}=\left(\frac{\boldsymbol{\omega}^{\mathrm{T}} \boldsymbol{\mu}_{j 1}}{\sum_{k=1}^{h} \boldsymbol{\omega}^{\mathrm{T}} \boldsymbol{\mu}_{j k}}, \frac{\boldsymbol{\omega}^{\mathrm{T}} \boldsymbol{\mu}_{j 2}}{\sum_{k=1}^{h} \boldsymbol{\omega}^{\mathrm{T}} \boldsymbol{\mu}_{j k}}, \cdots, \frac{\boldsymbol{\omega}^{\mathrm{T}} \boldsymbol{\mu}_{j h}}{\sum_{k=1}^{h} \boldsymbol{\omega}^{\mathrm{T}} \boldsymbol{\mu}_{j k}}\right)
$$


$u_{j k}=\frac{\boldsymbol{\omega}^{\mathrm{T}} \boldsymbol{\mu}_{j k}}{\sum_{k=1}^{h} \omega^{\mathrm{T}} \boldsymbol{\mu}_{j k}}, k=1,2, \cdots, h$

\section{Grade of Target Threat Assessment Method of Maximum Membership Degree}

In the grade of target threat assessment of the existing literature, often using maximum membership degree principle, namely the origin $u_{j \bar{k}}=\max _{1 \leq k \leq h}\left\{u_{j k}\right\}$ Belong to the first a threat level the evaluation target $T_{j}$.

A threat level level variables defined as $e_{k}(k=1,2, \cdots, h)$, the level of the target for determining value is:

$$
\begin{gathered}
v_{j}=(1,2, \cdots, h)\left(\mathrm{U}_{j}\right)^{\mathrm{T}}=\sum_{k=1}^{h} k u_{j k} \\
\text { s.t }\left\{\begin{array}{l}
u_{j k} \in[0,1] \\
\sum_{k=1}^{h} u_{j k}=1 \\
j=1,2, \cdots, n
\end{array}\right. \\
1=\sum_{k=1}^{h} u_{j k} \leq \sum_{k=1}^{h} k u_{j k} \leq \sum_{k=1}^{h} h u_{j k}=h
\end{gathered}
$$

In this type, $v_{j}$ is the number is a dimensionless index, levels between 1 (that is $e_{1}$ ) threats and between threat $h$, contains a comprehensive membership grade of target threat information and threat level 2. Because most of the time is not necessarily an integer, need further reasonable integer, says the biggest integer not greater than $v_{j}$, that is:

$$
\left[v_{j}\right]=k
$$

\begin{tabular}{|c|c|c|c|c|c|}
\hline target & $\mathrm{T}_{1}$ & $\mathrm{~T}_{2}$ & $\mathrm{~T}_{3}$ & $\mathrm{~T}_{4}$ & $\mathrm{~T}_{5}$ \\
\hline $\begin{array}{l}\text { The level } \\
\text { character }\end{array}$ & 1.9 & 2.0 & 2.4 & 2.9 & 3.3 \\
\hline $\begin{array}{l}\text { istic } \\
\text { value }\end{array}$ & 0 & 1 & 3 & 7 & 6 \\
\hline $\begin{array}{l}\text { Threat } \\
\text { level }\end{array}$ & 1 & 2 & 2 & 2 & 3 \\
\hline
\end{tabular}

Suppose you have 5 enemy air attack target $T_{j}(j=1,2, \cdots, 5)$, by type (2) the level of its decision value is calculated as listed in Table 1.

Table1 the enemy air attack target level decision value 


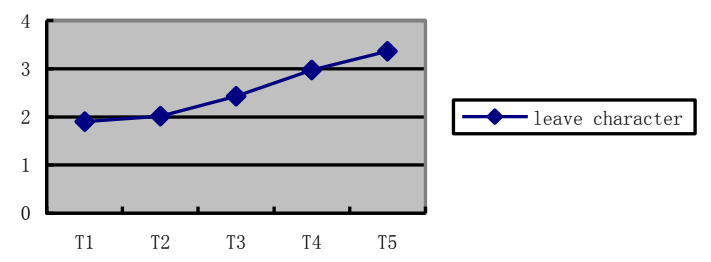

Figure 1. Enemy air attack target level decision value

Based on the principles of maximum membership degree obviously are: $\left[v_{1}\right]=1$, $\left[v_{2}\right]=\left[v_{3}\right]=\left[v_{4}\right]=2,\left[v_{5}\right]=3$

The conclusion that apparently do not agree with figure 1 intuitive judgment, put $v_{2}$ 、 $v_{3}$ and $v_{4}$ the corresponding target $T_{2} 、 T_{3}$ and threat assessment for level 2 is not reasonable. It is necessary in the evaluation of each batch of the grade of target threat, but also comprehensive considering the threat level in the degree of difference between each group of target.

\section{Binary Semantic Methods and Application}

A grade of target threat assessment method of binary semantic basic principle

Binary semantic concept [4][5][6] first appeared in the uncertainty decision problems, now apply it for grade of target threat evaluation. If $\operatorname{target} T_{j}(j=1,2, \cdots, n)$ level determine value is $v_{j} \in[1, h]$, the second yuan semantics can be represented as binary sequence groups $\left(e_{k}, \alpha_{j k}\right)$, and among them $e_{k} \in E$ and $\alpha_{j k} \in[-0.5,0.5)$, specific meaning can be described as: (1) $e_{k}$ is defined in advance good order in the "threat", said by level determination $E=\left\{e_{1}, e_{2}, \cdots, e_{h}\right\}$, and the most worth to close to the threat level; Transfer value $v_{j}$, (2) $\alpha_{j k}$ is symbol representation and for grade of target $T_{j}$ threat assessment results are obtained by the deviation of value (positive and negative respectively positive and negative deviation).

If $T_{j}(j=1,2, \cdots, n)$ level decision value meet the conditions:

$k-0.5 \leq v_{j}<k+0.5$

The assessment $T_{j}$ for the level of threat (that is $e_{k}$ ).

In order to depict the target belongs to the deviation degree of threat level, use deviation value is expressed as $\alpha_{j k}=v_{j}-k$.Clearly, $-0.5 \leq \alpha_{j k}<0.5$. If $\alpha_{j k} \in[-0.5,0)$ (that $\left|\alpha_{j k}\right|$ is smaller), the greater $v_{j} \in(k-1, k)$ the criterion, and the more close to $k$, its corresponding target $T_{j}$ by also belongs to the level of threat gradually reduced the threat level $k$. On the other hand, if the smaller $\alpha_{j k} \in(0,0.5)$, smaller $v_{j} \in(k, k+1)$, and the more close to $k$, the corresponding belongs to the greater threat level. If $a_{j k}=0$, then $v_{j}=k$, its corresponding right belongs to the level of the target $k$.

Using the concept of binary semantic explanation type (4): the level of target $T_{j}$ decision value represented as binary semantic $\left(e_{k}, \alpha_{j k}\right)$, which said the threat level 
assessment $e_{k},{ }^{\alpha}$ said, $v_{j}$ and by its deviation method for grade of target threat.

Using mathematical language, can be described as: $\tau:[1, h] \rightarrow E \times[-0.5,0.5)$ there is a reversible map making

$$
v_{j} \in[1, h] \mapsto \tau\left(v_{j}\right)=\left(e_{k}, \alpha_{j k}\right) \in E \times[-0.5,0.5), \quad k=\operatorname{Round}\left(v_{j}\right)
$$

Type, Rounded integral function or operator. Obviously, $\tau$ the inverse mapping $\tau^{-1}: E \times[-0.5,0.5) \rightarrow[1, h]$

$\left(e_{k}, \alpha_{j k}\right) \in E \times[-0.5,0.5) \mapsto \tau^{-1}\left(e_{k}, \alpha_{j k}\right)=v_{j} \in[1, h]$

Set $\left(e_{k}, \alpha_{j k}\right) 、\left(e_{l}, \alpha_{i l}\right)$ respectively is the target $T_{j}$ and $T_{i}$ the level of determination of the value, $v_{j} 、 v_{i}$ the binary semantic, provisions of its size is as follows:

(A)if $e_{k}>e_{l}$ (or $\left.k<l\right)$, then $\left(e_{k}, \alpha_{j k}\right)>\left(e_{l}, \alpha_{i l}\right)$;

(B)if $e_{k}=e_{l}$ (or $k=l$ ), then: (1) when $\alpha_{j k}=\alpha_{i l},\left(e_{k}, \alpha_{j k}\right)=\left(e_{l}, \alpha_{i l}\right)$; (2) when $\alpha_{j k}>\alpha_{i l}$, $\left(e_{k}, \alpha_{j k}\right)<\left(e_{l}, \alpha_{i l}\right)$; (3)when $\alpha_{j k}<\alpha_{i l}, \quad\left(e_{k}, \alpha_{j k}\right)>\left(e_{l}, \alpha_{i l}\right)$.

Accord of A condition if the grade of target threat is high, regardless of their deviation, its corresponding target threat level for determining value binary semantic. And Accord of B condition said in the same conditions, for grade of target threat target threat level for determining value binary semantic depends on the size of the deviation value, the smaller the deviation, the greater its corresponding binary semantic.

Then, according to the binary semantic $\left(e_{k}, \alpha_{j k}\right)$ threat level assessment and the threat of target $T_{j}(j=1,2, \cdots, n)$ in order. The specific methods and steps are as follows:

Step 1: using the type (5) to calculate the $\operatorname{target} T_{j}(j=1,2, \cdots, n)$ threat level for determining value $v_{j}$ binary semantic $\left(e_{k}, \alpha_{j k}\right)$;

Step 2: according to $\left(e_{k}, \alpha_{j k}\right)$ to determine all the batch of target threat level, and follow the order from large too small to determine the size of target threat sequencing, namely: the threat level is not at the same time, high threat level, its threat sorting at the top; Threat level at the same time, the deviation of small goals $\alpha_{j k}$, the threat sequencing at the top.

Bthe use of the grade of target threat assessment method of binary semantic instance

Using table 1 , according to the type (5) to calculate the target $T_{j}(j=1, \cdots, 5$ ) threat level for determining value $v_{j}$ binary semantic; Reusing binary semantic concepts and size relations, can be set for all grade of target threat and threats size sorting, as shown in Table 2.

Table 2 the enemy air attack target threat level assessment results and sorting

\begin{tabular}{|c|c|c|c|c|c|}
\hline target & $T_{1}$ & $T_{2}$ & $T_{3}$ & $T_{4}$ & $T_{5}$ \\
\hline decision value & 1.90 & 2.01 & 2.43 & 2.97 & 3.36 \\
\hline threat level by type (3) & 1 level & 2 level & 2 level & 2 level & 3 level \\
\hline binary semantic & $(2,-0.1)$ & $(2,0.01)$ & $(2,0.43)$ & $(3,-0.03)$ & $(3,0.36)$ \\
\hline $\begin{array}{l}\text { threat level from binary } \\
\text { semantic }\end{array}$ & 2 level & 2 level & 2 level & 3 level & 3 level \\
\hline $\begin{array}{l}\text { threat sorting from } \\
\text { binary semantic }\end{array}$ & 1 & 2 & 3 & 4 & 5 \\
\hline
\end{tabular}


As can be seen from Table 2, based on binary semantic evaluation and by type (3) for grade of target threat assessment is different, not only the conclusion in conformity with the principle of the previous analysis, more abundant and available information.

\section{Conclusion}

Based on binary semantic research shows that the grade of target threat evaluation model and method can not only determine the grade of target threat reasonably, can also distinguish the same threat level target threat sequencing, for automatic command and control system for grade of target threat assessment of symbolic computation provides a new scientific approach, to improve the level of operation supporting command decision and timeliness has important reference value. In addition, the grade of target threat assessment is essentially a special kind of multiple attribute (or goal, principles, factors and index) comprehensive evaluation (or decision) problem, so this paper established the grade of target threat assessment of binary semantic method can broaden the application to the multiple criteria group classification decision problems in other areas.

\section{References}

[1] Li Deng-feng. Status quo and progress directions of target threat assessment [J]. Systems Theory and Applications. 2009, 7(2): 1-9. (in Chinese)

[2] Zhao Fu-chang, Wang Rui, Zhang Na-wen, Bai Jian-Lin. The method of multi-sensor management based on rough entropy and target threat degree [J]. Journal of Air Force Engineering University (Natural Science Edition), 2009, 10(6): 28-31. (in Chinese)

[3] Wen Fei, Lv Yan, Duan Gang, Yu Min-jian. Application study of air-air threat assessment in synthetic decision aiding system [J]. Journal of System Simulation, 2009, 21(12): 243-246. (in Chinese)

[4] Herrera F, Herrera-Viedma E. Linguistic decision analysis: steps for solving decision problems under linguistic information [J]. Fuzzy Sets and Systems, 2000, 115(1): 67-82

[5] Herrera-Viedma E, Martínez L, Mata F. A consensus support system model for group decision-making problems with multigranular linguistic preference relations [J]. IEEE Transactions on Fuzzy Systems, 2005, 13(5): 644-658.

[6] Herrera F, Herrera-Viedma E, Martínez L. A fuzzy linguistic methodology to deal with unbalanced linguistic term sets [J]. IEEE Transactions on Fuzzy Systems, 2008, 16(2): 354-370.

[7] WANG Hong-jun, CHI Zhong-xian.Shipboard hard and soft weapon anti-missile decision-makingoptimization based on collaboration[J], Control and Decision, 2007, 22(3):299-303

[8] Liang Bo, Duan Ran.Fire distribution model based on feasible direction algorithm[J], Command Information System and Technology, 2013,4(2):30-32

[9] L Ozdamar. Emergency logistics planning in natural disasters [J]. Annal of Operation Research, 2004, 129(11): 218-219.

[10] Sarit Kraus.Negotiation and cooperation in mulfi-ageat environments.Artificial Intelligence. 1997: 79-97

[11] M.Ben-Bassat. Knowledge Requirement and Management in Expert Decision Support Systems for (Military) Situation Assessment [J]. IEEE Trans. on SMC.1982, 12(4):479-490 